\title{
Apoyo social percibido, autoestima y maternidad adolescente: entre el respeto y la intrusión. Estudio en Traiguén, Chile
}

\author{
Gloria Miryam Mora-Guerrero \\ Doctora en Estudios Americanos. Licenciada en Psicología \\ Universidad Católica de Temuco. Temuco, Chile \\ http://orcid.org/0000-0002-2391-4353・gmora@uct.cl
}

\section{Luisa Magdalena Escárate-Colín}

Licenciada en Psicología

Universidad Católica de Temuco. Temuco, Chile.

https:/ / orcid.org/0000-0003-1076-8091・pslescarate@gmail.com

\section{Carol Andrea Espinoza-Lerdón}

Licenciada en Psicología

Universidad Católica de Temuco. Temuco, Chile

https://orcid.org/0000-0003-2058-3832 • caroandreel.ps@gmail.com

\section{Andrea Beatriz Peña-Paredes}

Licenciada en Psicología

Universidad Católica de Temuco. Temuco, Chile

https:/ / orcid.org/0000-0001-6611-2118• andreapp.apa@gmail.com

Recibido: 20/11/2020 |Aprobado: 20/03/2021 |Publicado: 01/07/2021

Financiación o proveniencia del artículo: Fondo de financiamiento para invitación de expertos extranjeros por la Universidad Católica de Temuco, periodo de ejecución 2018-2019, código 2018 FIEE-GM-01. Colaboración metodológica: Mg. Jorge Dagoberto Constanzo Belmar.

\section{¿Cómo citar este artículo? / How to quote this article?}

Mora-Guerrero, G. M., Escárate-Colín, L. M., Espinoza-Lerdón, C. A., y Peña-Paredes, A. B. (2021). Apoyo social percibido, autoestima y maternidad adolescente: entre el respeto y la intrusión. Estudio en Traiguén, Chile. Prospectiva. Revista de Trabajo Social e intervención social, (32), 151-171. doi: 10.25100/prts.v0i32.10785. 


\section{Resumen}

Cuando la maternidad se presenta en la adolescencia, las mujeres requieren un sostén socioemocional que no siempre les pueden brindar sus familiares, pareja y personal de salud, lo que favorece que desarrollen una baja autoestima y conflictos con el rol materno. La investigación abordó este fenómeno explorando la relación entre apoyo social percibido, autoestima y maternidad a través de 15 entrevistas a mujeres que fueron madres adolescentes y cuyos hijos/as eran menores a 6 años. Los resultados mostraron que las participantes perciben el apoyo recibido en un continuo cuyas características van desde respetuoso hasta intrusivo o abiertamente dominante, lo que a su vez tiene implicaciones sobre su autoestima y autonomía materna. Las conclusiones alientan a repensar la maternidad adolescente como un evento vital que, a pesar de ser vivenciado con angustia, posibilita oportunidades para que las mujeres se desarrollen de manera integral, siempre y cuando cuenten con un apoyo social adecuado.

Palabras clave: Maternidad; Adolescencia; Apoyo social; Apoyo social percibido; Género. 


\title{
Perceived social support, self-esteem and teenage motherhood: respect and intrusion. Un study in Traiguen, Chile
}

\begin{abstract}
When motherhood occurs in adolescence, women require a socio-emotional support that their family, partner and health staff cannot always provide, which leads to the development of low self-esteem and conflicts with the maternal role. This research addressed this phenomenon by exploring the relationship between perceived social support, self-esteem and motherhood through 15 interviews with adolescent mothers whose children were under 6 years old. The results showed that the participants perceive the support received in a range which varies from respectful to intrusive or outright dominant, which in turn has implications for their self-esteem and maternal autonomy. The conclusions encourage the rethinking of adolescent motherhood as a vital event that, despite being experienced with anguish, provides opportunities for personal development for women if they have adequate social support.

Keywords: Maternity; Adolescence; Social support; Perceived social support; Gender.

Sumario: 1. Introducción, 2. Apoyo social percibido, autoestima y maternidad adolescente, 3. Metodología, 4. Hallazgos, 4.1. Apoyo social percibido: desde lo respetuoso a lo intrusivo/dominante, 4.2. Apoyo percibido y autoestima: seguridad e inseguridad, 4.3. Maternidad: posiciones para la autonomía o la no autonomía, 4.4 Apoyo ambivalente y maternidades paradójicas, 5. Conclusiones, 6. Referencias bibliográficas.
\end{abstract}




\section{Introducción}

La maternidad adolescente es una temática de amplia relevancia ya que, a nivel internacional, más de 16 millones de adolescentes tienen hijos cada año (Banco Mundial, 2014, Rodríguez, 2005, Rodríguez-Vignoli, Paez, Ulloa y Cox, 2017), mientras que en Chile en el 2016 un 15,2\% del total de los nacidos vivos fueron de mujeres entre 15 y 19 años (Dides et al., 2009), quienes constituyeron casi el 5\% del total de embarazos (Comisión Económica para América Latina y el Caribe [CEPAL], 2016), y, en el 2017, fueron responsables de 17.369 nacimientos, en 472 de ellos tratándose de madres menores de 15 años (Instituto Nacional de Estadística [INE], 2019). La maternidad en la adolescencia es un problema debido a que la mujer se encuentra en una etapa de profundos cambios biopsicosociales que implican inseguridad e incertidumbre y que, por tanto, favorecen la aparición de problemas como la depresión postparto y la baja autoestima (Borras-Santisteban, 2014, Chaj-Coyoy, 2015), además de que, con frecuencia, tiene lugar en condiciones de pobreza, lo que implica un factor de riesgo adicional para la salud de la joven y su hijo/a (González, González, Molina y Larrondo, 2019).

En efecto, en situación de vulnerabilidad socioeconómica, la experiencia materna facilita la aparición de problemáticas para las adolescentes, tales como complicaciones de salud y deserción escolar (Mazuera-Arias y Albornoz-Arias, 2017), dificultades para ejercer actividades remuneradas (Fondo de Población de las Naciones Unidas, 2014), complicaciones para reorganizar su sexualidad y fecundidad, y, establecer familias independientes (López-Gómez y Varela-Petito, 2016). Por estos motivos, las jóvenes requieren de un importante apoyo social que no siempre su familia, pareja y el personal médico puede brindarles, esto porque ellos mismos carecen de suficiente capacidad para dar contención y seguridad (López-Gómez y Varela-Petito, 2016), por lo que las adolescentes perciben que reciben poco sostén en el desempeño de su nuevo rol (RodríguezVignoli et al., 2017, Tubert, 1993).

La investigación se ubicó en este escenario para explorar la relación entre apoyo social, autoestima y maternidad, por medio de responder cómo percibieron las mujeres que fueron madres antes de los 18 años el apoyo social que recibieron y qué implicaciones tuvo dicho apoyo para su autoestima y maternidad. Se trató de una investigación cualitativa de tipo exploratorio que por medio de entrevistas indagó en la percepción del apoyo que recibieron por parte de sus familiares, pareja y el personal de salud 15 usuarias del programa para el Control del Niño Sano implementado en la ciudad de Traiguén, Región de la Araucanía, en el sur de Chile. 
La investigación se situó en el contexto de las políticas nacionales en salud que, desde hace al menos dos décadas, identificaron los derechos sexuales y reproductivos del adolescente como un asunto de salud pública. Así, en el 2000, los Objetivos Sanitarios para la década 2000-2010 incluyeron la prevención de embarazos en adolescentes (Ministerio de Salud, 2012), en 2008, la Política Nacional de Salud para Adolescentes y Jóvenes 2008-2015 comprometió a las instituciones a respetar sus derechos sexuales y reproductivos (Ministerio de Salud, 2008) y, ese mismo año, el programa Espacios Amigables obligó a los servicios de salud a garantizar para la población entre 10 y 19 años el derecho a ser atendido por personal capacitado (Ministerio de Desarrollo Social, 2014), en 2010, la Ley 20.418 estableció el derecho a la orientación para todas las madres (Ministerio de Salud, 2010), en 2012, el Programa Nacional de Salud Integral de Adolescentes y Jóvenes - Plan de Acción 2012-2020, priorizó las consejerías en salud sexual y reproductiva (Ministerio de Salud, 2012), en el 2015, los programas Espacios Amigables para Adolescentes y el Control Joven Sano fortalecieron la prevención y la promoción de la salud sexual y reproductiva adolescente, y, finalmente, en 2011, la Estrategia Nacional de Salud 2011-2020 fijó como meta que el 100\% de los establecimientos de atención primaria tuvieran un espacio amigable para los adolescentes (Ministerio de Salud, 2012).

En suma, la garantía de los derechos sexuales y reproductivos de la población juvenil se ha constituido en un objetivo prioritario y, en consecuencia, es necesario generar estudios que orienten la toma de decisiones a favor del respeto, la validación y el refuerzo incondicional de la experiencia materna, haciendo urgente reconstruir con esta finalidad las perspectivas de las jóvenes, con mayor razón si vivieron su maternidad en edades tempranas (Fernández y Manrique-Abril, 2010).

\section{Apoyo social percibido, autoestima y maternidad adolescente}

La adolescencia es un periodo donde tiene lugar un proceso de construcción de la identidad personal que se acompaña de sensaciones de invulnerabilidad y labilidad emocional, las cuales favorecen conductas de riesgo, como el comienzo de una vida sexual prematura (Ortiz-Torres, 2017, Rosabal-García, Romero-Muñoz, Gaquín-Ramírez y Hernández-Mérida, 2015) y la maternidad precoz (Maque-Ponce, Córdova-Ruíz, SotoRueda, Ramos-García y Rocano-Rojas, 2018). Cuando ésta se presenta, la mujer se ve cuestionada en sus expectativas de desarrollo personal (Jiménez, Aliaga y RodríguezVignoli, 2011) y, por lo general, se siente poco preparada para asumir su nuevo rol (ChajCoyoy, 2015). Por lo anterior, es fundamental el apoyo que percibe de sus familiares, de su pareja y del personal de salud en la medida que ella espera cuidados y apoyo emocional durante este momento de su vida (González et al., 2019). El apoyo que percibe es relevante porque contribuye a la construcción y la ratificación de su autoestima y aceptación personal, las que dependen fuertemente de la interiorización de la opinión de los demás (Bravo, 
Mora-Guerrero, Escárate-Colín, Espinoza-Lerdón y Peña-Paredes

Contreras, Dois, Contreras y Rojas, 2017, González et al., 2019). Por esto, contar con apoyo social, se constituye como un factor protector de la adolescente en tanto promueve que tenga mayor seguridad para ejercer la maternidad y desarrollar conductas que aseguren una buena calidad de vida para ella y su hijo/a (Asociación Argentina de la Prevención de Violencia familiar, 1998, González et al., 2019, González, Orcasita, Carrillo y Palma-García, 2017, Lavielle-Sotomayor et al., 2014).

A pesar de la relevancia que puede tener el apoyo social, las familias y los equipos sanitarios pueden manifestar sentimientos de rechazo, sorpresa y /o frustración hacia la maternidad de la joven (Moscoso-Álvarez, Rodríguez-Figueroa, Reyes-Pulliza y Colón, 2016, Rangel, Valerio, Patiño y García, 2004), lo que genera que ésta sienta que sus redes de apoyo no la contienen, sino que más bien le hacen dudar de sus capacidades y le dificultan las tareas maternas (Bravo et al., 2017). Complementariamente, si la joven está inmersa en dinámicas familiares jerárquicas, de dependencia y/o sometimiento a las mujeres con base en normas de género, o bien, a los/las adolescentes con base en normas adulto-centristas, el apoyo que recibe se acompaña de otras actitudes que merman su independencia, autonomía y capacidad decisiva (Frydenberg y Lewis, 1997, González et al., 2019).

Dinámicas de esta naturaleza también tienen lugar de parte del personal de salud que puede brindar un trato no empático (Denno, Hoppes \& Chandra-Mouli, 2015) o restar capacidad de decisión a la mujer (Rodríguez-Vignoli, 2011, Rojas-Ramírez, EguigurenBravo, Matamala-Vivaldi, Palma-Manríquez y Gálvez-Pérez, 2017). Este tipo de relaciones, donde las jóvenes son infantilizadas, son producto de lógicas que estereotipan a las adolescentes como indefensas, frágiles y desamparadas que requieren depender de un adulto (Busso, 2001, Dides \& Fernández, 2016, Miranda-Palacios, 2018, Mori-Quispe et al., 2015,) y que, por tanto, promueven en ellas el desarrollo de una baja autoestima y sentimientos de inseguridad como madres (Bravo et al., 2017, Rodríguez-Vignoli, 2011, Rodríguez-Vignoli et al., 2017).

La presente investigación asume la teoría del apoyo social como marco analítico del problema de estudio y, en tanto, define el apoyo social como una práctica simbólico-cultural que incluye los principales vínculos de la persona, los que favorecen que ésta se adapte a situaciones de estrés, se motive al cambio y al planteamiento de nuevas metas (Aranda y Pando, 2013, Guzmán, Hakkert, Contreras y Falconier, 2002). El apoyo social incluye la expresión de afecto positivo de una persona hacia la otra, la afirmación o respaldo de sus comportamientos, percepciones o puntos de vista, así como la entrega de ayuda material o simbólica (Frey, 1989). En esta investigación, el apoyo social representa un factor protector para que la condición de madre adolescente no se convierta en un obstáculo para la vida de la joven ni en un riesgo para su salud (Castro, Campero y Hernández, 1997, Hupcey \& Morse, 1997, Reina-Barreto, Criollo-Espín y Fernández-D’ Andrea, 2019). 
El apoyo social tiene su contraparte en el apoyo social percibido, es decir, en el punto de vista personal de la ayuda material y/o simbólica que se recibe (Sarason, Shearin, Pierce \& Sarason, 1987). El apoyo social percibido refiere a la interpretación particular que hace cada persona del apoyo que recibe, conforme a sus expectativas, necesidades, su forma de recibir, entregar y significar las acciones, así como las oportunidades que tenga para conversar, aclarar dudas y tener contención (Sarason et al., 1987). Bajo esta perspectiva, en esta investigación, se concibe el apoyo social percibido por las madres adolescentes, como las aportaciones de tipo emocional, material, informacional y de compañía que ellas perciben de parte de los miembros de su red social (Gracia-Fuster, Herrero-Olaizola y Musitu-Ochoa, 2002). En el apartado siguiente se presenta la metodología del estudio y, en seguida, los resultados y la discusión.

\section{Metodología}

Se trató de una investigación de enfoque cualitativo, con el propósito de explorar la relación entre apoyo social percibido, autoestima y maternidad desde el punto de vista interaccional de mujeres que fueron madres en su adolescencia (Vega-Malagón et al., 2014). Bajo esta directriz, se conformó una muestra de jóvenes que experimentaron la maternidad antes de los 18 años y que, al momento de participar de la investigación, eran usuarias del Hospital Dino Stagno de la ciudad de Traiguén, en la región de la Araucanía, a donde acudían al Programa Chile Crece Contigo (ChCC) para realizar el Control del Niño Sano de su hijo/a. Dicho programa da acceso a todas las usuarias del sistema de salud público a servicios de acompañamiento, protección y apoyo de matronas, enfermeras y médicos especialistas desde el primer control del embarazo hasta el ingreso del niño/a al sistema escolar (Chile Crece Contigo, s.f.).

A fin de comprender el contexto geográfico de las participantes, se describe que, según el Instituto Nacional de Estadísticas (INE, 2019), la ciudad de Traiguén, al año 2017, contaba con aproximadamente 18.843 habitantes, de los cuales 9.824 eran mujeres y 4.957 se encontraba en situación de pobreza, mientras que el 32,3\% de los hogares también se encontraba en esta situación y en 2.123 casos se trataba de familias mononucleares con mujer jefa de hogar. Respecto a datos de maternidad, en 2016, el 11,9\% de los nacimientos fue de mujeres de 15 a 19 años, y, el 56,9\% de los nacimientos provinieron de mujeres con estudios medios, el $25,9 \%$ de mujeres con educación superior y el 16,7\% con estudios básicos (Universidad de La Frontera, 2020).

El tipo de muestreo fue no probabilístico de tipo homogéneo, lo que significó trabajar con mujeres de perfil similar en cuanto a situación socioeconómica y vivencias personales (Hernández-Sampieri, Fernández-Collado y Baptista-Lucio, 2010). Las participantes fueron 15 mujeres entre 18 a 24 años, que estuvieron embarazadas durante su adolescencia. Todas 
Mora-Guerrero, Escárate-Colín, Espinoza-Lerdón y Peña-Paredes

reportaron que habían sido madres entre los 14 a los 17 años, en 6 de los 15 casos, la participante tenía pareja (pololo), y, en 11 casos, vivía con sus padres. Ninguna estaba casada. Se obtuvieron más datos para caracterizar la muestra a partir de una entrevista exprofeso a un profesional del área de maternidad del Hospital Dino Stagno, quien mencionó que el perfil socioeconómico y de salud de las usuarias incluye descriptores como: pertenencia a familias de niveles socioeconómicos medio a medio bajo, presencia de disfuncionalidad familiar o violencia intrafamiliar, patrones de relación de pareja esporádicas, caracterizadas por la exploración sexual, la práctica de relaciones sexuales sin protección, codependencia y violencia en el pololeo, consumo exploratorio de alcohol y drogas, y, sintomatología depresiva anterior al embarazo. La muestra excluyó a mujeres que habían sido madres como producto de una vulneración sexual.

En cuanto a las técnicas de recolección de información, las participantes respondieron a una entrevista semiestructurada e individual donde se les invitó a conversar sobre su autoestima y toma decisiones en torno a la maternidad durante los primeros años de vida de su hijo/a, sobre todo en relación con el apoyo que sintieron de parte de figuras familiares significativas, su pareja actual y/o anterior, y el personal de salud, en específico, enfermeras y matronas del Control del Niño Sano. Adicionalmente, se tomaron notas de campo donde se registraron las vivencias personales y transferencias de la persona entrevistadora. Cada entrevistada firmó un consentimiento informado donde se garantizaron sus derechos como informante.

El análisis de datos fue realizado según la teoría fundamentada (Bérnard-Calva, 2016, Strauss y Corbin, 2002). Se utilizó el software Atlas.ti versión 7.5 para llevar a cabo la codificación abierta, la codificación axial y la codificación selectiva. Como explica Kelle (2005), la teoría fundamentada permite distinguir dos tipos de códigos: los sustantivos, generados de modo inductivo a partir de la comparación de los datos empíricos, y, los teóricos, que permiten combinar los códigos sustantivos por medio del conocimiento teórico previo. De acuerdo con estos lineamientos, en la Tabla 1 se presenta el proceso de conformación de las categorías y subcategorías, mientras que en la Figura 1 se muestra el modelo teórico final. Cabe señalar que, para garantizar la validez del estudio, se realizó una triangulación de instrumentos, por medio de codificar por separado las entrevistas y las notas de campo y, posteriormente, contrastar ambos análisis. 
Mora-Guerrero, Escárate-Colín, Espinoza-Lerdón y Peña-Paredes

Tabla 1. Proceso de conformación de categorías y subcategorías.

\begin{tabular}{|c|c|c|c|}
\hline $\begin{array}{l}\text { Categoría } \\
\text { central }\end{array}$ & $\begin{array}{c}\text { Categorías } \\
\text { relacionadas }\end{array}$ & Subcategorías & $\begin{array}{c}\text { Códigos abiertos } \\
\text { (ejemplos) }\end{array}$ \\
\hline Códig & teóricos & Códig & sustantivos \\
\hline \multirow{6}{*}{$\begin{array}{l}\text { Maternidad } \\
\text { adolescente }\end{array}$} & \multirow[t]{2}{*}{$\begin{array}{l}\text { Apoyo social } \\
\text { percibido }\end{array}$} & Amoroso / Respetuoso & $\begin{array}{c}\text { Otorgando relevancia a la } \\
\text { retroalimentación materna } \\
\text { Sintiendo apoyo de la } \\
\text { pareja actual }\end{array}$ \\
\hline & & Intrusivo / Dominante & Sintiéndose juzgadas \\
\hline & \multirow[b]{2}{*}{ Autoestima } & Seguridad emocional & Autovaloración personal \\
\hline & & Inseguridad emocional & $\begin{array}{c}\text { Necesidad de ser } \\
\text { reconocidas por otro/a } \\
\text { Desestimación en vivencias } \\
\text { personales }\end{array}$ \\
\hline & \multirow[b]{2}{*}{ Autonomía } & $\begin{array}{l}\text { Toma de decisiones } \\
\text { basada en sí misma }\end{array}$ & Crecimiento personal \\
\hline & & $\begin{array}{c}\text { Toma de decisiones } \\
\text { basada en la búsqueda } \\
\text { de aceptación por } \\
\text { otros/as }\end{array}$ & $\begin{array}{c}\text { Toma de decisiones por } \\
\text { presión social }\end{array}$ \\
\hline
\end{tabular}

Fuente: elaboración propia.

\section{Hallazgos}

Los resultados permiten reconocer a la maternidad adolescente como un fenómeno relacional en donde la joven toma a la figura proveedora de apoyo como un referente para construir un sentimiento de valía y seguridad personal desde el cual puede afianzarse en la toma de decisiones sobre la crianza. No obstante, las jóvenes otorgan significados al apoyo que reciben en modos que se entienden a partir de un continuo positivo a negativo de acuerdo con las relaciones en las cuales están insertas. De esta manera, cuando están inmersas en relaciones basadas en el respeto, tienden a percibir el apoyo que reciben - sea este de tipo informacional, emocional o material - como amoroso, así como a vincularlo con el desarrollo de su autoestima y maternidad.

De igual modo, cuando se insertan en relaciones jerárquicas, usualmente basadas en prácticas y normas tradicionales de género o adulto-céntricas, lo más común es que perciban el apoyo recibido como intrusivo, dominante e, incluso, violento, y que lo asocien con inseguridad personal y limitaciones en su capacidad de decisión como madres. En otras palabras, comprender la percepción que tienen las adolescentes respecto del apoyo social 
Mora-Guerrero, Escárate-Colín, Espinoza-Lerdón y Peña-Paredes

que reciben cuando son madres implica partir de su posición mayor o menormente igualitaria o jerárquica frente a los/as otros/as y considerar las alternativas que ellas construyen tanto para valorarse a sí mismas como para ejercer la crianza y proyectarse más allá de su rol materno. Las relaciones entre apoyo social percibido, autoestima y maternidad se presentan en la Figura 1 y se detallan enseguida.

Figura 1. Apoyo social percibido, autoestima y maternidad adolescente.

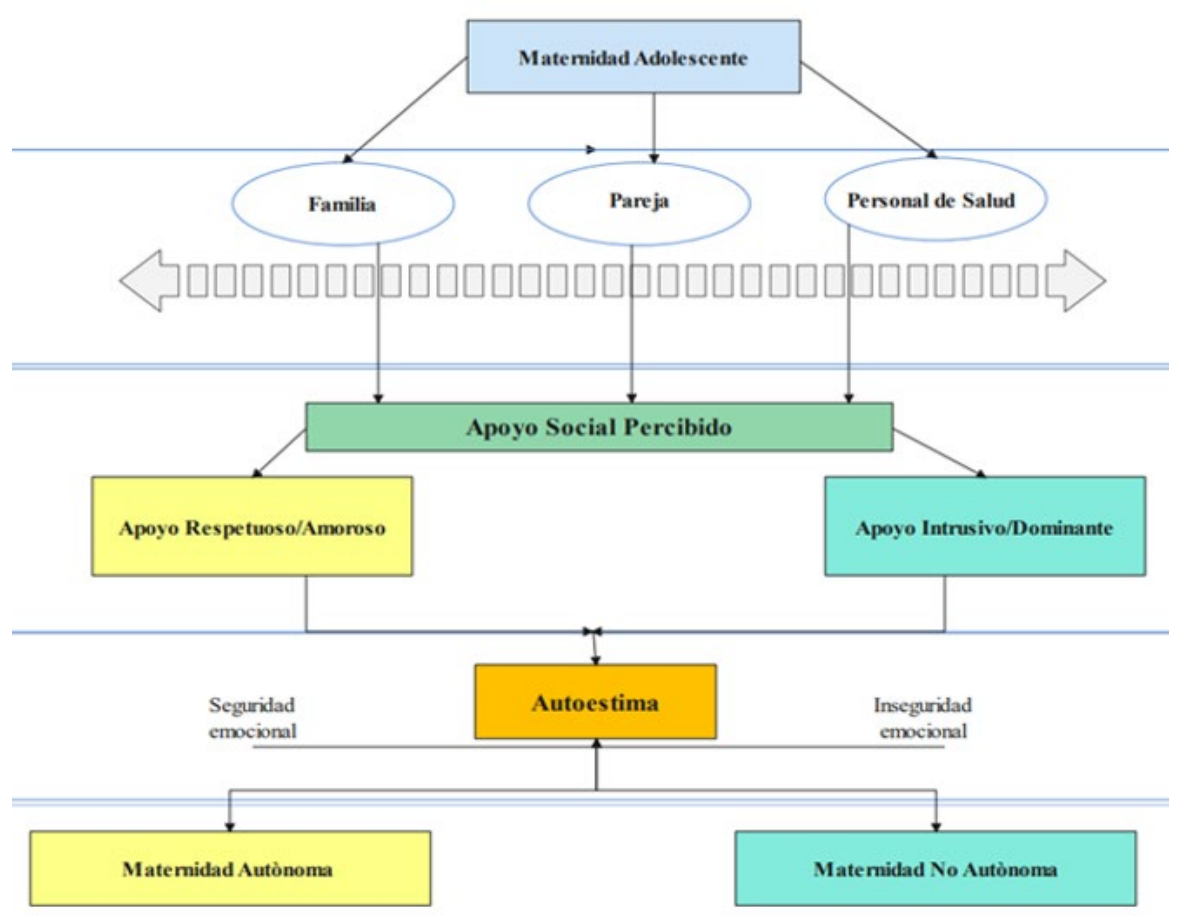

Toma de decisiones

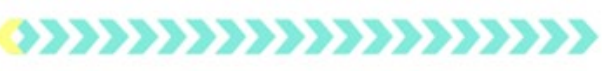

Fuente: elaboración propia.

\subsection{Apoyo social percibido: desde lo respetuoso a lo intrusivo/ dominante}

A modo global, las entrevistadas sienten que reciben un apoyo mayormente respetuoso o intrusivo e, incluso, dominante o violento, dependiendo de si la figura proveedora de apoyo les abre o no espacio para la toma de decisiones sobre la crianza. Así, refieren que sus figuras familiares más significativas les brindan un apoyo respetuoso cuando demuestran que aceptan su maternidad, mientras que les ofrecen uno intrusivo cuando las critican o desautorizan en su nuevo rol. Como dijo una participante: "Es difícil ser mamá a veces, me cansa harto, yo trato de poner normas, en la casa todos los adultos me desautorizan, no puedo tomar decisiones con mi hijo" (entrevistada n³, 24 años), con lo que deja entrever un contexto familiar de orden adulto-céntrico donde se siente desautorizada como madre. 
Mora-Guerrero, Escárate-Colín, Espinoza-Lerdón y Peña-Paredes

Las informantes otorgan gran relevancia al apoyo que les pueden brindar las figuras masculinas. Comentan que, si sus padres asumen un rol paterno en la educación del niño/a, sobre todo cuando el padre biológico de este último está ausente, ellas se sienten respaldadas y experimentan un sentimiento de bienestar. Esto se nota en el siguiente relato: "Mi papá me ayuda harto, se queda con él, lo va a dejar al jardín... Su figura paterna es él" (entrevistada $n^{\circ} 5,21$ años). Sin embargo, las figuras paternas también pueden brindan este $\mathrm{u}$ otros apoyos a la par que critican o descalifican a sus hijas, frecuentemente, basándose en estereotipos de género que prevalecen al interior de las familias. Se nota lo anterior en el siguiente comentario: “iMi papá se enojó [cuando se enteró del embarazo]! Habló con mi pololo y lo retó. Se enojó conmigo hasta que tenía seis meses. Me decía que él tenía que preocuparse de mí, porque era su obligación como hombre” (entrevistada n², 24 años).

En su rol como nuevas madres, las adolescentes tienen importantes expectativas del apoyo que sus propias madres o abuelas puedan proporcionarles. Las jóvenes esperan que estas últimas las acompañen y orienten, pero sin interferir con sus decisiones y siendo capaces de mirarlas no solo como madres, sino también como estudiantes, trabajadoras, futuras profesionales o, simplemente, mujeres. Una joven menciona: “Yo le pido opiniones [a mi mamá], digo: 'mamá pasa esto', me da su opinión y ahí veo si la considero, es importante porque [ella] me enseñó todo de ser mamá" (entrevistada n ${ }^{\circ}$ 14, 23 años). Sin embargo, las madres no siempre están en condiciones de apoyar de esa manera disputando, a juicio de las adolescentes, el rol de madres. Así lo indica con frustración una entrevistada: “Mi mamá a veces me dice: ¡Eso no es así!', trata a mi hija como si fuera de ella, eso me insecuriza" (entrevistada $\mathrm{n}^{\mathrm{o}} 12,21$ años).

Tratándose de las relaciones de pareja, las adolescentes viven un apoyo respetuoso y amoroso en primer lugar cuando su pareja, actual o pasada, asume el rol paterno frente al/la hijo/a. Una entrevistada comenta: "Mi pololo, a pesar de que éramos niños, ha sido el pilar fundamental de esta familia, siempre está para mí y nuestro hijo" (entrevistada n¹5, 21años). Las adolescentes que viven una relación con estas características refieren que se sienten comprendidas, que confían en su pareja y que esto les ayuda a generar un vínculo más satisfactorio con su bebé. Se destacó el relato de una participante que fue madre por segunda vez: “Es diferente sentirse y estar sola con un bebé, me enojaba con mi hija [refiere a su primera experiencia como madre], ahora me siento acompañada [con su pareja actual] y trato de ser mejor mamá con mis bebés y con ella [su primera hija], la escucho como lo hacen conmigo" (entrevistada n5, 24 años).

En un contexto cultural donde se mantienen los roles tradicionales de género, la relación de pareja se vuelve un lugar donde las jóvenes sienten que reciben sostén, no obstante, que sigue siendo común que la pareja las controle o, yendo más allá, las violente. Bajo estas relaciones, la joven expresa un fuerte malestar emocional que asocia a la falta de contención y acompañamiento en la crianza por parte de la pareja, como se observa en este 
Mora-Guerrero, Escárate-Colín, Espinoza-Lerdón y Peña-Paredes

comentario: "Me sentía mal [con mi pololo], tenía una necesidad que debía suplir con su afecto (...) No era sano, porque, me hacía sufrir, pero yo lo necesitaba" (entrevistada n³, 23 años). Es muy posible que convertirse en madre durante la adolescencia sea un factor de riesgo para que las mujeres permanezcan en patrones de relación dominantes o abusivos. En efecto, de acuerdo con los testimonios, las jóvenes tienen una importante necesidad de afecto y validación por otros, lo que es esperable en esta etapa evolutiva, pero que sumado a la amenaza común de abandono por parte del padre del/la bebé, les genera ansiedad y una mayor necesidad de apoyo que, en el mejor de los casos, sus familias nucleares pueden soslayar.

El apoyo mayor o menormente respetuoso o intrusivo lo refieren las participantes también en lo que respecta al personal de salud. Algunas mencionan que han recibido una atención respetuosa de parte de enfermeras y matronas quienes les han brindado contención y cuidado, como se observa en esta entrevista: "La matrona me ayudaba, estaba pendiente de mí y lo que pasaba en mi familia, si tenía las cosas para poder ser mamá" (entrevistada n 7,23 años). En este punto destaca la demanda de la joven por ser mirada en su salud no sólo física, sino también psicológica y social. Lamentablemente, las participantes comentan que también han sido discriminadas por parte del personal de salud, de nueva cuenta con base en prejuicios de género o adulto-céntricos. Una entrevistada comenta: “La matrona me daba miedo... A veces uno se queda callada... Me dijo: ¡Aguántatelas eso te pasó por abrir las piernas!" (entrevistada nº 15, 21 años).

El malestar generalizado y la vivencia de discriminación que las adolescentes sienten de parte del personal sanitario, lo relacionan con el hecho de que prefieren evitar la asistencia a este tipo de servicios. Es decir, cumplen con los controles obligatorios para el/la bebé, pero no acuden a talleres de maternidad, lactancia, primeros cuidados y controles de fertilidad. Una participante comenta: “[Las enfermeras] te decían cómo vestir a tu bebé, y opinaban, uno se sentía cuestionada y al final no daban ganas de ir, ni a los controles con la matrona" (entrevistada no8, 21 años). Es posible que el alejamiento de los programas sanitarios tuviera efectos sobre la salud sexual de algunas adolescentes, así, tres de las quince participantes fueron madres por segunda vez antes que su primer hijo cumpliera cuatro años. Por otra parte, el apoyo social inadecuado por parte del personal aumenta las inequidades en salud, ya que no todas las mujeres pueden pagar una atención permanente en el sistema particular. En el caso de las entrevistadas, nueve de quince había acudido a un servicio privado cuando menos en una ocasión por su desconfianza frente a la atención pública. 
Mora-Guerrero, Escárate-Colín, Espinoza-Lerdón y Peña-Paredes

\subsection{Apoyo percibido y autoestima: seguridad e inseguridad}

En la experiencia de las entrevistadas, el apoyo que reciben tiene implicaciones sobre su autoestima en la medida que les favorece el desarrollo de sentimientos de seguridad e/o inseguridad. Es decir, cuando perciben un apoyo respetuoso, se genera en ellas una sensación de mayor confianza en sus capacidades por lo que se sienten con recursos personales para validarse a sí mismas. En cambio, cuando perciben un apoyo intrusivo, se sienten más inseguras y con mayor necesidad de buscar la aprobación de terceras personas para validarse como madres. Aunque estas relaciones no son taxativas, las adolescentes sí marcan en sus discursos que un apoyo positivo, aunado a una mayor seguridad, las motiva a buscar estrategias para su desarrollo integral, por ejemplo, a encontrar espacios recreativos y de autocuidado. Sobre este aspecto, dice una participante: "Yo me doy tiempo para mí, porque hay cosas que uno hace más que ser mamá, y también, con mi hijo para estar fuera de la casa y entendernos mejor" (entrevistada n³, 24 años).

En cambio, percibir un apoyo mayormente intrusivo hace más posible que las jóvenes se sientan disminuidas en su autoestima, tal como expresa una entrevistada con tono de cansancio respecto a su relación de pareja: “Los primeros meses me sentía mal, el papá de mi hija me reprimía. Mi autoestima bajó mucho". Otra joven manifiesta constantes dudas de sí misma: “Mis papás se ponen en contra de mí, me desautorizan [en la crianza], me frustra y me hace dudar si lo hago bien [como madre]" (entrevistada nº, 20 años). Dicho de otra forma, cuando las informantes perciben un apoyo negativo, son más vulnerables frente a las críticas y tienen por lo general menos oportunidades en sus relaciones sociales para afirmarse a sí mismas. Una tercera participante agrega: “Después uno se acostumbra a que le digan cosas [agresivas], una señora me dijo: ¡Te jodiste la vida! [por el embarazo], y ahí no decía nada" (entrevistada ${ }^{\circ} 10,23$ años). De este modo, el apoyo social que la madre adolescente percibe funciona como un factor mediador de su maternidad, es decir, tiene consecuencias sobre su autoestima y seguridad personal y, a su vez, a mayor o menor autoestima, ella tiene más o menos posibilidades de vivir una maternidad autónoma.

\subsection{Maternidad: posiciones para la autonomía o la no autonomía}

De acuerdo con las informantes, un apoyo social mayormente respetuoso favorece que desarrollen una maternidad más segura y autónoma, es decir, que tengan más capacidad para decidir sobre la crianza y, en general, sobre sus vidas. En cambio, un apoyo social en gran medida intrusivo favorece que vivan su maternidad bajo una mayor influencia de terceras personas, en la búsqueda de una validación externa muy anudada en su rol materno. 
Mora-Guerrero, Escárate-Colín, Espinoza-Lerdón y Peña-Paredes

Las participantes comentan que una maternidad autónoma significa sentirse responsable en la crianza y adecuada para responder a las necesidades del/la bebé, lo que a su vez aumenta su seguridad personal. Ellas manifiestan que una experiencia materna en estos términos incluye espacios para el enriquecimiento del vínculo madre-hijo/a, así como sentirse con aptitud para la educación infantil. El testimonio siguiente da cuenta de este aspecto: “¡No! Las decisiones [en relación con mi hijo] las tomo yo, por lo que yo considero, no pienso en lo que [otros] me van a decir" (entrevistada n³, 23 años). Es decir, cuando las jóvenes se posicionan desde este lugar tienen suficiente seguridad para ejercer su maternidad, sin necesidad de buscar la validación externa para ejercer la crianza.

Desde esta posición las jóvenes también son capaces de hacer del apoyo percibido un factor potenciador de su identidad integral. Entonces manifiestan tener planes a futuro construidos desde múltiples roles, por ejemplo, proyectan independizarse de su núcleo familiar, tener una casa propia y desempeñarse laboralmente. Al respecto, una participante menciona: "Tomé la decisión de tener una carrera profesional para darle un mejor futuro a mi hija" (entrevistada n6, 21 años). Este tipo de decisiones desarrollan en las adolescentes un sentimiento de orgullo que se apoya en el ejercicio del rol materno. Dicho en otras palabras, posicionadas desde una maternidad autónoma, en relaciones idealmente no jerárquicas con el mundo adulto o de pareja, las mujeres ingresan en un círculo virtuoso en el que el apoyo social que perciben se traduce en mejor autoestima, ésta, en mayor autonomía para la maternidad, y, esta última, en un factor retroalimentador de su validación personal.

Por el contrario, las informantes asocian el apoyo social intrusivo con maternidades presionadas por juicios de terceros, sobre exigencia emocional y un sentimiento de limitación de su libertad. Una entrevistada comenta: "Mi papá manda en la casa, al estar con ellos, tengo que adaptarme y hacer caso, y mi hija también, es como su hija, no la mía" (entrevistada $\mathrm{n}^{\circ} 1,24$ años), lo que evidencia que debido a su dependencia económica se ve en la obligación de adaptarse a las exigencias familiares. Con una incomodidad similar, otra participante menciona: “Tengo que responder en todo estudiando, darle mayor estabilidad a mi hijo y demostrarle a mis papás que puedo, aun cuando haya cometido un error [el embarazo]" (entrevistada nº, 24 años). Además, las informantes relacionan el apoyo intrusivo con una dificultad para posicionarse en roles distintos al materno, dicho en palabras de una de ellas: "Mi mamá me dice: 'Cómprate esto' y yo prefiero comprarle a mi hija. Me ofrecieron trabajo, pero cuido a mi hija, también dejé la posibilidad de estudiar, por dedicarme a ella". 
Mora-Guerrero, Escárate-Colín, Espinoza-Lerdón y Peña-Paredes

En síntesis, si bien la maternidad puede ser un ejercicio autónomo en la adolescencia y una posición para el crecimiento integral, también puede ser un lugar en el cual la joven esté sometida a una fuerte presión de terceras personas de quienes, paradójicamente, espera recibir un apoyo significativo. En este sentido los resultados mostraron que el apoyo social que perciben las participantes tiene una doble faceta y que, por tanto, sus implicaciones tienen dos lecturas ya sea que favorezcan en alguna medida (o no) el desarrollo de la seguridad personal y la maternidad autónoma.

\subsection{Apoyo ambivalente y maternidades paradójicas}

El estudio exploró la relación entre el apoyo social percibido, la autoestima y la maternidad, desde el punto de vista de mujeres que fueron madres en su adolescencia. Los resultados alientan la hipótesis de que, cuando las mujeres perciben un apoyo respetuoso, mejoran su autoestima y, por consiguiente, desarrollan más autonomía como madres, mientras que, cuando perciben un apoyo intrusivo, se favorece un sentimiento de disminución en su valía y seguridad personal, así como un sentimiento de presión y limitación en su rol materno (Rodríguez-Vignoli et al., 2017). En este sentido, los hallazgos son consistentes con la teoría del apoyo social percibido, que enfatiza la importancia de las redes cercanas para el desarrollo de la identidad y la autoestima adolescente (Bravo et al., 2017).

Dicho en otras palabras, los testimonios recolectados permiten generar información a partir de la cual describir el fenómeno relacional del apoyo social percibido en su tránsito hacia lo intrapersonal, dando lugar a imaginarios subjetivos en las jóvenes, para quienes la interiorización de las opiniones externas es parte importante en la ratificación de su valía personal (Padrós-Blázquez, Gutiérrez-Hernández y Medina-Calvillo, 2015). Desde otra perspectiva, los resultados muestran la articulación entre percepción de apoyo social, autoestima y toma de decisiones sobre la crianza teniendo relevancia a nivel teórico porque se insertan en una discusión contemporánea donde los conceptos científicos, como apoyo social percibido y maternidad, aluden a fenómenos comprensibles en relaciones ambivalentes (Kelly, 2017).

De hecho, los vínculos humanos adquieren una doble faceta, donde una misma persona puede generar sentimientos de acompañamiento $\mathrm{y}$, a la vez, de limitación de la autonomía. En la experiencia de las madres adolescentes, el apoyo que reciben del entorno puede implicar un soporte informacional o material y, en paralelo, un cuestionamiento a su valía o competencia (Bravo et al., 2017), esto último porque el/la proveedor/a de apoyo no responde a las necesidades de quien recibe la ayuda, o bien, porque la ayuda tiene lugar en una relación asimétrica, con frecuencia marcada por jerarquías sociales de género o adultocéntricas (González et al., 2019). 
Mora-Guerrero, Escárate-Colín, Espinoza-Lerdón y Peña-Paredes

Algo similar ocurre con el concepto de maternidad, el que se concreta en prácticas situadas histórica, social y económicamente que requieren ser problematizadas e incluso desnaturalizadas para encontrar respuestas a favor de la equidad y el bienestar subjetivo de las mujeres (Losantos-Velasco, Montoya-Caero, Exeni-Ballivian, Santa Cruz-Terrazas y Loots, 2016). Así, en este estudio las participantes continuaron la lógica de género que asocia la maternidad al sacrificio (Armijo-Garrido, 2016, Giallorenzi, 2017), lo que se observó principalmente cuando se encontraron involucradas en vínculos que experimentaron como intrusivos y que implicaron una limitación a su rol materno y una búsqueda de validación externa. Empero, posicionadas desde otro lugar, para las jóvenes informantes la maternidad fue motor para el desarrollo de su autoestima y su identidad, aún si el embarazo había ocurrido en circunstancias desfavorables como la temprana edad y la carencia de recursos económicos. Se puede decir que las maternidades adolescentes son fenómenos paradójicos, con cargas limitantes y angustiantes, pero también posibilidades de nuevas metas para el desarrollo, en especial, si se cuenta con un apoyo social respetuoso.

Los resultados alientan a los equipos de salud a generar intervenciones que promuevan el bienestar de las adolescentes considerando su género, edad y nivel socioeconómico. Asimismo, se recomienda que la atención en salud adolescente se refuerce en calidad por medio de sensibilizar al personal respecto a la importancia de que brinde un apoyo social respetuoso y oriente a las figuras significativas de las madres adolescentes para que puedan ofrecerles un apoyo amoroso e incondicional. Por último, es necesario pensar en programas de apoyo económico que permita que estas jóvenes puedan contar con un mínimo nivel de vida a partir del cual estén en condiciones de establecer relaciones de base material más igualitarias y, por tanto, de apoyo mutuo con sus seres cercanos.

Desde el punto de vista metodológico se destaca que los hallazgos son ricos en descripciones relativas a la maternidad, la autoestima y la identidad de las participantes. Se recomienda reproducir el estudio con mujeres que al momento del trabajo de campo estén cursando el embarazo o el puerperio y sean menores de 18 años. Esto permitirá precisar los resultados y comprender si se vieron influidos por la maduración psicobiológica de las entrevistadas.

\section{Conclusiones}

Los resultados mostraron que las maternidades son prácticas situadas histórica, social y económicamente y que, por tanto, su comprensión requiere de una mirada particular y pertinente a cada mujer. Tratándose de la mujer madre adolescente, los estudios son coincidentes cuando describen una experiencia materna comúnmente inmersa en contextos de pobreza y dinámicas familiares y de articulación institucional jerárquicas, de dependencia y/o sometimiento (González et al., 2019, Rojas-Ramírez et al., 2017). Bajo esta lógica, las investigaciones concuerdan que la experiencia materna en edades tempranas 
Mora-Guerrero, Escárate-Colín, Espinoza-Lerdón y Peña-Paredes

conlleva riesgos para el bienestar biopsicosocial de la joven madre y su hijo/a (LópezGómez y Varela-Petito, 2016, Mazuera-Arias y Albornoz-Arias, 2017).

En este escenario, la investigación desarrollada mostró un panorama alternativo: aquel que proviene de la reconstrucción de las subjetividades juveniles, producto de relaciones menor o mayormente inequitativas, donde con base en prejuicios de género o adulto-centristas, se justifica la dependencia de las jóvenes (Dides y Fernández, 2016, Miranda-Palacios, 2018), pero donde ellas se dan a la tarea de proponer para sí mismas y sus entornos cercanos otras significaciones maternas. Así, hacen de la maternidad un evento vital que, a pesar de ser vivenciado con angustia, se integra a sus vidas en la búsqueda de opciones para el desarrollo de una identidad integral y un planteamiento hacia el futuro.

Las madres adolescentes, en su demanda simultánea de apoyo y autonomía, tienen la posibilidad de deconstruir lógicas de género y adulto-céntricas. Es responsabilidad de los equipos científicos contribuir a la generación de instancias donde estas disputas puedan reconfigurarse en conocimientos con potencial de impacto a las familias, el personal en salud y la sociedad en general para que se tome conciencia del papel que pueden jugar las mujeres jóvenes, si se potencia su desarrollo por medio de brindarles un apoyo social basado en el respeto hacia la autonomía materna. La ciencia social y de la salud puede generar alternativas contextualizadas para la comprensión de los significados socioemocionales de la maternidad de las adolescentes, pudiendo favorecer la transformación de su posición social a partir del reconocimiento de las necesidades y proyecciones contextuales, afectivas y relacionales de las mujeres.

\section{Referencias bibliográficas}

Aranda, C., y Pando, M. (2013). Conceptualización del apoyo social y las redes de apoyo social. Revista de Investigación en Psicología, 16(1), 233-245. doi: 10.15381/rinvp.v16i1.3929.

Armijo-Garrido, L. (2016). Ciudadanía y cuidado en España: el dilema trabajo/familia según las madres trabajadoras. Psicoperspectiva, 15(3), 87-100. doi: 10.5027/ psicoperspectivas-Vol15Issue3-fulltext-789.

Asociación Argentina de Prevención de la Violencia Familiar. (1998). Manual de capacitación y recursos para la prevención de la violencia familiar. Buenos Aires, Argentina: Asociación Argentina de Prevención de la Violencia Familiar.

Banco Mundial. (2014). Tasa de fertilidad en adolescentes (nacimientos por cada 1.000 mujeres entre 15 y 19 años de edad). Recuperado de http:/ / datos.bancomundial.org/indicador/SP.ADO.TFRT.

Bérnard-Calva, S. (Coord.). (2016). La teoría fundamentada: una metodología cualitativa. México: Universidad Autónoma de Aguascalientes. 
Mora-Guerrero, Escárate-Colín, Espinoza-Lerdón y Peña-Paredes

Borras-Santisteban, T. (2014). Adolescencia: definición, vulnerabilidad y oportunidad. Correo Científico, Médico, 18(1), 05-07. Recuperado de http:/ / scielo.sld.cu/scielo.php?script=sci_arttext\&pid=S1560-43812014000100002.

Bravo, P., Contreras, A., Dois, A., Contreras, M., y Rojas, A. (2017). Necesidades psicosociales y características de una intervención online para apoyar a las madres adolescentes. Revista chilena de obstetricia y ginecología, 82(5), 566-573. doi: 10.4067/s0717-75262017000500566.

Busso, G. (junio, 2001). Vulnerabilidad social: nociones e implicancias de políticas para América Latina y el Caribe a comienzos del Siglo XXI. En Seminario Internacional sobre las diferentes expresiones de la vulnerabilidad social en América Latina y el Caribe. CEPAL/CELADE, Santiago, Chile.

Castro, R., Campero, L., y Hernández, B. (1997). La investigación sobre apoyo social en salud: situación actual y nuevos desafíos. Revista de Saúde Pública, 31(4), 425-435.

Chaj-Coyoy, G. (2015). Adaptación emocional en el embarazo precoz (Trabajo de pregrado). Universidad Rafael Landívar. Quetzaltenango, Guatemala.

Comisión Económica para América Latina y el Caribe [CEPAL]. (2016). Horizontes 2030: la igualdad en el centro del desarrollo sostenible. Santiago, Chile: Naciones Unidas.

Denno, D., Hoppes, A., \& Chandra-Mouli, V. (2015). Effective strategies to provide adolescent sexual and reproductive health services and to increase demand and community support. Journal of Adolescent Health, 56(1), 22-41. doi: 10.1016/j.jadohealth.2014.09.012.

Dides, C., Benavente, C., Guajardo, A., Undurraga, J., Sáez, I., y Castellón, M. (2009). Embarazo en la Adolescencia en Chile. Santiago, Chile: Aprofa. Unfpa. Flacso.

Dides, C., y Fernández, C. (2016). Primer Informe Salud Sexual Salud Reproductiva y Derechos Humanos En Chile. Embarazo adolescente (Vol. 2). Recuperado de http://mileschile.cl/wpcontent/uploads/2017/04/Informe-DDSSRR-2016-Cap\%C3\%ADtulo-II.pdf.

Fernández, A. R., y Manrique-Abril, F. G. (2010). Efecto de la intervención educativa en la agencia de autocuidado del adulto mayor hipertenso de Boyacá, Colombia, Suramérica. Ciencia y Enfermería, 16(2), 83-97. Recuperado de https://scielo.conicyt.cl/pdf/cienf/v16n2/art_09.pdf.

Frey, M. (1989). Social support and health: A theoretical formulation derived from King's Conceptual Framework. Nursing Science Quarterly, 2(3), 138-148. doi: 10.1177/089431848900200309.

Frydenberg, E., y Lewis, R. (1997). ACS: Escalas de Afrontamiento para Adolescentes (Adaptación española de J. Perena y N. Seisdedos). Madrid, España: TEA Ediciones.

Fondo de Población de las Naciones Unidas. (2014). Programa de Acción. Aprobado en la Conferencia Internacional sobre la Población y el Desarrollo. El Cairo, 5 a 13 de septiembre de 1994. Edición 20 Aniversario. Recuperado de https://www.un.org/en/development/desa/population/publications/ICPD_prog ramme_of_action_es.pdf.

Giallorenzi, M. L. (2017). Crítica feminista sobre la noción de la buena madre. Revista reflexiones, 96(1), 87-95. Recuperado de https:/ / dialnet.unirioja.es/descarga/articulo/6124752.pdf.

Gobierno de Chile. (s.f.). Chile Crece Contigo. Recuperado de http://www.crececontigo.gob.cl/. González, V., Orcasita, L., Carrillo, J. P., y Palma-García, D. (2017). Comunicación familiar y toma de decisiones en sexualidad entre ascendientes y adolescentes. Revista 
Mora-Guerrero, Escárate-Colín, Espinoza-Lerdón y Peña-Paredes

Latinoamericana de Ciencias Sociales, Niñez y Juventud, 15(1), 419-430. doi: 10.11600/1692715x.1512605022016.

González, E., González, D., Molina, T., y Larrondo, P. (2019). Variables familiares, nivel socioeconómico y apoyo social asociadas a comportamientos de riesgo en salud en adolescentes chilenos. Cuadernos Médico Sociales, 59(2), 23-35.

Guzmán, J. M., Hakkert, R., Contreras, J. M., y Falconier, M. (2002). Diagnóstico sobre la salud reproductiva de jóvenes y adolescentes en América Latina y el Caribe. México: Fondo de Población de las Naciones Unidas.

Gracia-Fuster, E., Herrero-Olaizola, J., y Musitu-Ochoa, G. (2002). Evaluación de recursos y estresores psicosociales en la comunidad. Madrid: Síntesis.

Hernández-Sampieri, R., Fernández-Collado, C., y Baptista-Lucio, P. (2010). Metodología de la investigación (5 ed.). México: McGraw Hill.

Hupcey, J. E., \& Morse, J. M. (1997). Can a professional relationship be considered social support? Nursing outlook, 45(6), 270-276. doi: 10.1016/s0029-6554(97)90006-3.

Instituto Nacional de Estadística [INE]. (2019). Número de nacimientos en Chile descendió 5,4\% entre 2016 y 2017. Recuperado de https://www.ine.cl/prensa/2019/11/15/n\%C3\%BAmerode-nacimientos-en-chile-descendi\% C3\% B3-5-4-entre-2016-y-2017.

Jiménez, M. A., Aliaga, L., y Rodríguez-Vignoli, J. (2011). Una mirada desde América Latina y el Caribe al objetivo de Desarrollo del Milenio de acceso universal a la salud reproductiva. Santiago: Naciones Unidas. Celade.

Kelle, U. (2005). ¿Hacer "emerger" o "forzar" los datos empíricos? Un problema crucial de la teoría fundamentada reconsiderada. Forum Qualitative Sozialforschung / Forum: Qualitative Social Research, 6(2), Art. 27. doi: 10.17169/fqs-6.2.467.

Kelly, C. (2017). Care and violence through the lens of personal support workers. International Journal of Care and Caring, 1(1), 97-113. doi: 10.1332/239788217X14866305589260.

Lavielle-Sotomayor, P., Jiménez-Valdez, F., Vázquez-Rodríguez, A., Aguirre-García, M. C., Castillo-Trejo, M., y Vega-Mendoza, S. (2014). Impacto de la familia en las conductas sexuales de riesgo de los adolescentes. Revista Médica del Instituto Mexicano del Seguro Social, 52(1), 38-43.

López-Gómez, A., y Varela-Petito, C. (Coords.). (2016). Maternidad en adolescentes y desigualdad social en Uruguay. Análisis territorial desde la perspectiva de sus protagonistas en barrios de la periferia crítica de Montevideo. Montevideo: Universidad de la República-UDELAR. UNFPA.

Losantos-Velasco, M., Montoya-Caero, T., Exeni-Ballivian, S., Santa Cruz-Terrazas, M., y Loots, G. (2016). Aplicando la Epistemología Socioconstruccionista a la Investigación en Psicología. International Journal of Collaborative Practice, 6(1), 32-46.

Maque-Ponce, M. L., Córdova-Ruíz, R. L., Soto-Rueda, A. M., Ramos-García, J. M., y RocanoRojas, L. (2018). Embarazo no planeado: impacto de los factores socioculturales y emocionales de la adolescente, 2017. Investigación Valdizana, 12(2), 83-94. doi: 10.33554/riv.12.2.143.

Mazuera-Arias, R., y Albornoz-Arias, N. (2017). Maternidad adolescente, desigualdad social y exclusión educativa en el Norte de Santander (Colombia) y Táchira (Venezuela). Espacio abierto. Cuaderno venezolano de sociología, 26(1), 121-137. 
Mora-Guerrero, Escárate-Colín, Espinoza-Lerdón y Peña-Paredes

Ministerio de Desarrollo Social. (2014). Espacios Amigables. Informe de Seguimiento de Programas Sociales (cierre al 31 - Diciembre - 2014). Santiago: Gobierno de Chile. Recuperado de http:/ / www.programassociales.cl/pdf/2015/PRG2015_3_59205.pdf.

Ministerio de Salud. (2008). Política Nacional de Salud de Adolescentes y Jóvenes. Santiago, Chile: Ministerio de Salud de Chile. Recuperado de https://www.minsal.cl/wpcontent/uploads/2015/09/Pol\%c3\%adtica-Nacional-de-Salud-de-Adolescentes-yJ\%c3\%b3venes-2008-2015-Chile.pdf.

Ministerio de Salud. (2010). Objetivos Sanitarios para la Década 2000-2010. Evaluación de final del período. Santiago: Gobierno de Chile.

Ministerio de Salud. (2012). Programa Nacional de Salud Integral de Adolescentes y Jóvenes. Plan de Acción 2012-2020. Nivel primario de atención. Santiago, Chile: Gobierno de Chile. Recuperado de https://www.minsal.cl/portal/url/item/d263acb5826c2826e04001016401271e.pdf.

Miranda-Palacios, R. (2018). Directrices de Salud para implementar programas de educación de la sexualidad, con visión personalista, para adolescentes - Perú. Apuntes de bioética, (1)1, 44-56. doi: 10.35383/apuntes.v1i1.189.

Mori-Quispe, E., Contreras-Pulache, H., Hinostroza-Camposano, W. D., Lam-Figueroa, N., Huapaya-Huertas, O., Chacon-Torrico, H. ... Urrutia-Aliano, D. (2015). Evaluación de un instrumento para cuantificar la estigmatización de la adolescente embarazada en el Perú. Anales de la Facultad de Medicina, 76(2), 141-146. doi: 10.15381/anales.v76i2.11140.

Moscoso-Álvarez, M., Rodríguez-Figueroa, L., Reyes-Pulliza, J., y Colón, H. (2016). Adolescentes de Puerto Rico: una mirada a su salud mental y su asociación con el entorno familiar y escolar. Revista Puertorriqueña de Psicología, 27(2), 320-332. Recuperado de https:/ / www.redalyc.org/pdf/2332/233247620009.pdf.

Ortiz-Torres, M. F. (2017). Conductas de riesgo y factores psicosociales en madres adolescentes. San Juan Ostuncalco, Quetzaltenango, Guatemala, julio 2016 (Trabajo de pregrado). Guatemala: Universidad Rafael Landívar.

Padrós-Blázquez, F., Gutiérrez-Hernández, C. Y., y Medina-Calvillo, M. A. (2015). Propiedades psicométricas de la Escala de Satisfacción con la Vida (SWLS) de Diener en población de Michoacán (México). Avances en Psicología Latinoamericana, 33(2), 221-230. doi: 10.12804/apl33.02.2015.04.

Rangel, J., Valerio, L., Patiño, J., y García, M. (2004). Funcionalidad familiar en la adolescente embarazada. Revista de la Facultad de Medicina UNAM, 47(1), 24-27. Recuperado de https://www.medigraphic.com/cgi-bin/new/resumen.cgi?IDARTICULO=646.

Reina-Barreto, J. A., Criollo-Espín, C. A., y Fernández-D’ Andrea, K. (2019). El apoyo social en la maternidad indeseada de estudiantes universitarias ecuatorianas: Análisis desde la perspectiva de género. Prospectiva. Revista de Trabajo Social e intervención social, (27), 107137. doi: $10.25100 /$ prts.v0i27.6745.

Rojas-Ramírez, G., Eguiguren-Bravo, P., Matamala-Vivaldi, M. I., Palma-Manríquez, I., y Gálvez-Pérez, T. (2017). Acceso a anticoncepción en adolescentes: percepciones de trabajadores de la salud en Huechuraba, Chile. Revista Panamericana de Salud Pública, 41, e7. doi: 10.26633/RPSP.2017.77. 
Mora-Guerrero, Escárate-Colín, Espinoza-Lerdón y Peña-Paredes

Rodríguez, J. (2005). Reproducción en la adolescencia: el caso de Chile y sus implicaciones de política. Revista de la Cepal, 86, 123-146. Recuperado de https://repositorio.cepal.org/bitstream/handle/11362/11072/086123146_es.pdf?seque nce $=1 \&$ isAllowed $=y$.

Rodríguez-Vignoli, J. (2011). Reproducción adolescente y desigualdades: VI encuesta nacional de juventud, Chile. Revista Latinoamericana de Población, 5(8), 87-113.

Rodríguez-Vignoli, J., Paez, K., Ulloa, C., y Cox, L. (2017). Reproducción en la adolescencia en Chile: la desigualdad continúa y urgen políticas activas. Santiago, Chile: CEPAL. UNFPA. Recuperado de https://www.cepal.org/es/publicaciones/41135-reproducción-laadolescencia-chile-la-desigualdad-continúa-urgen-políticas.

Rosabal-García, E., Romero-Muñoz, N., Gaquín-Ramírez, K., y Hernández-Mérida, R. A. (2015). Conductas de riesgo en los adolescentes. Revista Cubana de Medicina Militar, 44(2), 218-229. Recuperado de http://scielo.sld.cu/scielo.php?script=sci_arttext\&pid=S013865572015000200010\&lng=es\&tlng=es.

Sarason, B., Shearin, E., Pierce, G., \& Sarason, I. (1987). Interrelations of social support measures: Theoretical and practical implications. Journal of Personality and Social Psychology, 52(4), 813-832. doi: 10.1037/0022-3514.52.4.813.

Strauss, A., y Corbin, J. (2002). Bases de la investigación cualitativa. Técnicas y procedimientos para desarrollar la teoría fundamentada. Medellín, Colombia: Universidad de Antioquia.

Tubert, S. (1993). Demanda de hijo y deseo de ser madre. Debate Feminista, (8), 349-355.

Universidad de La Frontera. (2020), Actualización PLADECO. Plan de Desarrollo Comunal Traiguén 2020-2024. Araucanía, Chile: Universidad de la Frontera. Recuperado de https:/ / mtraiguen.cl/wp-content/uploads/2020/01/INFORME-PLADECO-

Versi \%c3\%b3n-Resumen-ejecutivo-Concejo-y-WEB.pdf.

Vega-Malagón, G., Ávila-Morales, J., Vega-Malagón, A. J., Camacho-Calderón, N., BecerrilSantos, A., y Leo-Amador, G. E. (2014). Paradigmas en la investigación. Enfoque cuantitativo y cualitativo. European Scientific Journal, 10(15), 523-528. 


\section{OTROS ARTÍCULOS DE PROSPECTIVA No. 32 DE 2021}

\section{EDITORIAL}

Compromisos, dilemas y desafíos del Trabajo Social con dimensión colectiva en tiempos de pandemia

Enrique Pastor-Seller

\section{ARTÍCULOS}

La narrativa biográfica como alternativa para la comprensión del Conflicto y la violencia en Colombia: una experiencia pedagógica universitaria

Sara Sofía Castaño-Barco y Claudia BermúdezPeña

Experiencias de transición a la vida adulta de jóvenes que migraron solos. Un estudio en Aragón (España) Daniel Jiménez-Franco; Raquel Berzosa-Callén y Chabier Gimeno-Monterde

Fundamentals of Education in Intervention with Young Offenders. A Critical Review of a 'Successful' Case in Andalucía, España

David Herrera-Pastor

Subjetividad juvenil: lecturas desde y para el Trabajo Social en Colombia

Daniela Joya-Valbuena

Participación politica de los jóvenes del Valle del Cauca, Colombia, en la toma de decisiones públicas

Luis Eduardo Gutiérrez-Rojas y Amanda Ramírez-Giraldo

Victimización y perpetración de violencia en pareja adolescente y redes de apoyo en Colombia. Análisis con perspectiva de género

Johanna Alexandra Reina-Barreto
Apoyo social percibido, autoestima y maternidad adolescente: entre el respeto y la intrusión. Estudio en Traiguén, Chile

Gloria Mora-Guerrero, Luisa Escárate-Colín, Carol Espinoza-Lerdón y Andrea Peña-Paredes

El desarrollo en el Trabajo Social de Colombia: un campo transversal, disperso y polivalente

Maira Judith Contreras-Santos

Análisis de la situación laboral de las alimentadoras en la caficultura de la zona central colombiana, a la luz de la teoría de Nancy Fraser

Pablo Andrés Arango-Giraldo

Prioridades de investigación en la Obra social de empleados públicos, Mendoza, Argentina. Un estudio Delphi sobre producción de conocimientos en la seguridad social

Cecilia Amalia Molina

Producción de conocimiento y toma de decisiones. Relaciones entre academia y política pública para las familias en Colombia

José Raúl-Ruíz y Sandro Leonardo MunévarVargas

El staff de prácticas profesionales en Trabajo Social: espacio de fomento del aprendizaje autodirigido. Un estudio de caso en la Universidad Católica Luis Amigó, Colombia

Cristian Eduardo Blanco-García

\section{ARTISTA INVITADO}

Margie Reinel-Aguilar Ao Cicadidae

Prospectiva

\section{PROSPECTIVA Revista de Trabajo Social e Intervención Social}

No. 32 • jul.-dic. 2021

e-ISSN: 2389-993X • Universidad del Valle 